\title{
EIMERIOSIS EN CRÍAS DE ALPACAS: PREVALENCIA Y FACTORES DE RIESGO
}

\author{
Eimeriosis in Young Alpacas: Prevalence and Risk Factors
}

Antony Rodríguez H. ${ }^{1}$, Eva Casas A. ${ }^{2}$, Luis Luna E. ${ }^{2}$, César Gavidia Ch. ${ }^{2}$, Víctor Zanabria H. ${ }^{3}$, Raúl Rosadio A. ${ }^{1,2,4}$

\section{Resumen}

Se analizaron 478 muestras fecales de crías (1-90 días de edad), aparentemente saludables, en el Centro de Investigación y Producción (CIP - La Raya) de la Universidad Nacional del Altiplano, Puno. Las muestras fueron procesadas mediante la técnica de sedimentación y flotación (solución saturada de azúcar) buscando ooquistes y por el método de McMaster para determinar la carga parasitaria (ooquistes por gramo de hecesOPGH). En 418/478 (87.5\%) de las muestras se detectaron ooquistes de Eimeria spp (24,017 OPGH, IC 7,534; rango: 50-1'202,400), preferentemente E. lamae (60.4\%), E. macusaniensis (50.4\%), E. alpacae (45.6\%), E. punoensis (30\%) y E. ivitaensis (6.24\%). La infección parasitaria se incrementó con la edad. Inicialmente se encontró en el 50\% de 24 muestras de 1-30 días de edad (17,216 OPGH), y luego de 93\% de 82 crías de 31-45 días (28,501 OPGH), 85\% de 144 crías de 46-60 días (34,731 OPGH), 94\% de 183 crías de 61-75 días (16,564 OPGH) y 80\% de 45 crías de 76-90 días (17,376 OPGH). Las infecciones por $E$. lamae se detectaron muy tempranamente (41\% en muestras de 1-30 días) alcanzando tasas de 66.7\% (46-60 días), mientras que E. macusaniensis se inicia en el 4.2\% de crías de 1-30 días y alcanza la máxima infección (65.6\%) en el grupo de 61-75 días. En el 28.2 y $28.2 \%$ de las muestras positivas se detectaron infecciones dobles y triples, respectivamente, y en el 11.2\% se encontró cuatro especies de Eimeria. En las asociaciones dobles predominó la coexistencia de E. lamae y E. alpacae y de E. lamae y E. macusaniensis.; en las asociaciones triples predominaron E. alpacae, E. lamae y E. macusaniensis y en las cuádruples se encontró E. punoensis, E. alpacae, E. lamae y E. macusaniensis. El análisis de regresión logística multivariada mostró que los rangos de edad de 1-30 días (OR=0.19, IC 95\%: 0.08-0.48), 61 a 75 días (OR=2.49, IC95\%: 1.22-5.04) y la separación de las crías (OR=0.2, IC95\%: 0.08-0.48) estuvieron asociados a la infección de Eimeria spp.

Palabras clave: eimeriosis, crías, alpacas, infecciones múltiples, E. lamae, E. macusaniensis

${ }^{1}$ CONOPA - Instituto de Investigación y Desarrollo de Camélidos Sudamericanos, Lima

${ }^{2}$ Facultad de Medicina Veterinaria, Universidad Nacional Mayor de San Marcos, Lima

${ }^{3}$ Facultad de Veterinaria y Zootecnia, Universidad Nacional del Altiplano de Puno, Puno

${ }^{4}$ E-mail: rrosadio@gmail.com 
A total of 478 fecal samples taken from healthy alpaca crias aged 1-90 days at Centro de Investigación y Producción (CIP - La Raya), Universidad Nacional del Altiplano, Puno, were processed by the sedimentation and flotation technique in saturated sugar solution to detect oocysts and by the McMaster method to determine oocysts per gram (OPG) of fecal sample. Eimeria spp were present in 418 (87.5\%) of the samples with a mean of 24,017 OPG (CI of 7,534 and range 50-1,202,400). The most frequent species were E. lamae (60.4\%), E. macusaniensis (50.4\%), E. alpacae (45.6\%), E. punoensis (30\%), and E. ivitaensis (6.24\%). Parasite load increased with age, 50\% of 24 samples at 1-30 days were positive with an average load of 17,216 OPG, at 31-45 days 93\% of 82 (28,501 OPG), at $46-60$ days $85 \%$ of 144 ( 34,731 OPG), at $61-75$ days $94 \%$ of $183(6,564$ OPG) and at $76-90$ days $80 \%$ of 45 with 17,376 OPG. E. lamae comprised $41 \%$ of all coccidian oocysts at 1-30 days and the highest rate of infection (66.7\%) was reached at 46-60 days, whereas E. macusaniensis was present in only $4.2 \%$ of crias at 1-30 days, but peaked (65.6\%) at 61-75 days. Double, triple, and quadruple infections were found in 28.2, 28.2, and 11.2\% of positive samples respectively. The coexistence of E. lamae with E. alpacae and, E. lamae with $E$. macusaniensi infection were common in double infections, whereas $E$. lamae, E. alpacae and E. macusaniensis were mainly found in triple infections, and $E$. lamae, E. alpacae, E. punoensis and E. macusaniensis in quadruple infections. The multivariate logistic regression analysis showed that the age range of 1-30 days $(\mathrm{OR}=1.19$, CI 95\%: 0.08-0.48), 61-75 days (OR=2.49, CI 95\%: 1.22-5.04) and separation of offsprings (OR=0.2, CI 95\%: 0.08-0.48) were associated with Eimeria spp infection.

Key words: eimeriosis, crias, alpacas, eimeria co-infections, E. lamae, E. macusaniensis

\section{INTRODUCCIÓN}

Los camélidos sudamericanos (CSA) constituyen un recurso genético de importancia económica, social, cultural y científica para el Perú y para los países de la región andina. El término CSA incluye a dos especies domésticas: la alpaca (Vicugna pacos) y la llama (Lama glama), y dos silvestres: la vicuña (Vicugna vicugna) y el guanaco (Lama guanicoe); distribuidos lo largo del trapecio andino entre los 3600 y los $5500 \mathrm{msnm}$ (Wheeler, 1995). En estos pisos altitudinales, las especies domésticas constituyen el único medio de subsistencia para un vasto sector rural, proveyendo fundamentalmente de fibra y carne a la población (FAO, 2005), en tanto que las especies silvestres son igualmente un importante potencial genético y pecuario para el aprovechamiento sustentable por parte de las comunidades campesi- nas, dentro de los marcos legales establecidos para el uso de estas especies (Wheeler, 1995).

La mayor población de alpacas se encuentra en el Perú (90\%), y la zona de Puno es la primera región productora de alpacas en el país con el 58\% de la población nacional (1'681,919) (FAO, 2005). Alrededor del 90\% de las alpacas peruanas está en manos de pequeños productores y comunidades campesinas que, paradójicamente, constituyen uno de los segmentos menos favorecidos de la población y que sobreviven en estado de extrema pobreza (FAO, 2005). En estos lugares, la crianza de la alpaca se lleva a cabo siguiendo sistemas productivos tradicionales y carentes de tecnologías adecuadas, que se traducen en alta morbilidad y mortalidad neonatal, baja natalidad y un pobre desarrollo productivo, que inciden negativamente en la producción de carne y fibra. 
En estas condiciones, las enfermedades parasitarias ocasionan pérdidas cuantiosas a los productores, las cuales se estiman en un millón y medio de dólares anuales (Leguía, 1991; Leguía y Casas, 1999). Los animales se encuentran expuestos a diversas parasitosis, incluyendo los endoparásitos, que les causan trastornos intestinales ocasionando disminución del apetito, baja conversión alimenticia y anemia (Melo y Hurtado, 1985; Leguía, 1991; Rosadio y Ameghino, 1994; Palacios et al., 2006).

La eimeriosis o coccidiosis, afecta principalmente a las crías, causando infecciones subclínicas durante los primeros tres meses de vida, con prevalencias de 30 al 100\% (Leguía y Casas, 1999). Estudios recientes muestran una mayor agresividad patogénica dentro del complejo diarreico neonatal, llegando a ser causantes de infecciones fatales, particularmente en crías que en campo se registran como mortalidades por "diarrea", colibacilosis, e incluso con cuadros asociados a enterotoxemia (Rosadio y Ameghino 1994; Palacios et al., 2006; Rosadio et al., 2010).

En el Perú, se disponen de estudios que describen las especies de eimerias que afectan a las alpacas, así como información sobre prevalencia y patogenicidad (Guerrero, 1967; Rosadio y Ameghino, 1994; Leguía y Casas 1999); sin embargo, muchos de los aspectos epidemiológicos han sido deducidos o extrapolados de otras especies de animales. Por este motivo, el objetivo del presente estudio fue determinar la prevalencia y conocer los factores de riesgo que influyen en la presentación de eimerias en crías de alpacas en una granja experimental de Puno.

\section{Materiales y Métodos}

Las muestras coprológicas fueron tomadas en el Centro de Investigación y Producción (CIP - La Raya) de la Universidad Nacional del Altiplano de Puno, distrito de
Santa Rosa, Puno, que mantiene una población de 4000 alpacas en una extensión de 6000 hectáreas, a una altura de 4136 a 5470 msnm, y con temperatura media de $6.6{ }^{\circ} \mathrm{C}$ $\left(-10\right.$ a $\left.17^{\circ} \mathrm{C}\right)$.

La crianza es de tipo extensiva, basada en pastoreo sobre pastos naturales. Los animales se encuentran agrupados en "majadas" o "puntas" de parición, hembras vacías, machos reproductores y tuis (jóvenes). Durante la etapa de parición, cada "punta” de 250 a 300 madres, se subdivide en un grupo compuesto por madres con crías nacidas durante la primera mitad de la campaña (finales de diciembre a mitad de febrero) y otro de madres con crías nacidas en la segunda mitad de la campaña (quincena de febrero hasta finalizar la campaña). La granja lleva registros de parición y empadre, mortalidad y producción.

Se colectaron muestras de heces a 478 crías (1-90 días de edad), de 13 grupos provenientes de 6 "puntas" de parición que mantenían crías separadas en los dos subgrupos etarios y de una "punta” donde no se realizó la subdivisión etaria.

Las muestras fecales fueron obtenidas durante la segunda quincena de marzo de 2008. Las muestras de heces, tomadas al azar, fueron obtenidas directamente del recto utilizando bolsas de polietileno como guantes, rotulando procedencia, número de arete, raza, edad y sexo de las crías. Las muestras fueron guardadas en refrigeración y se trasladaron al Laboratorio de Parasitología de la Facultad de Medicina Veterinaria, Universidad Nacional Mayor de San Marcos, Lima.

Las muestras fueron procesadas mediante los métodos de sedimentación y flotación en solución saturada de azúcar, para determinar la presencia de ooquistes, y por el método de Mc Master en similares soluciones saturadas, para determinar la carga parasitaria y diferenciar las especies de eimerias, siguiendo la metodología descrita por Leguía y Casas (1999). 
En las muestras positivas a ooquistes de Eimeria spp se volvió a tomar una parte de la muestra inicial para homogenizarla y tamizarla a fin de eliminar la materia fecal gruesa. La suspensión fecal tamizada, conteniendo los ooquistes, fue depositada en copas de precipitación para obtener el sedimento y decantar el sobrenadante. El sedimento resultante fue transferido a recipientes de vidrio donde se adicionó una solución de bicromato de potasio al 2.5\% para inhibir el crecimiento bacterial y promover la esporulación a temperatura ambiente. La identificación de las especies de Eimeria se hizo en base a las características morfológicas y tamaño del ooquiste, usando un ocular micrométrico, de acuerdo a descripciones existentes (Guerrero, 1967; Guerrero et al., 1970a; Leguía y Casas, 1999).

Para el análisis estadístico, los datos se agruparon de acuerdo a la presencia o ausencia de ooquistes de coccidias en las muestras de heces, teniendo en cuenta como factores de riesgo la edad de las crías (rangos de 1-30, 31-45, 46-60, 61-75 y 76-90 días), bofedales (presencia o ausencia), práctica de separación de crías (crías mayores y menores versus no separación), ubicación de las “canchas" de parición (zonas altas y bajas), sexo (macho y hembra) y raza (Huacaya y Suri). Posteriormente, con las variables consideradas de riesgo significativo se realizó un modelo multivariado eligiendo el de mayor probabilidad. El análisis de los datos se hizo a través de una regresión logística utilizando el paquete estadístico STATA v 10.0, buscando determinar los "Odds ratio" (OR). Los resultados fueron expresados con intervalos de confianza al 95\%. Las variables con valores $\mathrm{p}<0.05$ fueron consideradas significativas.

\section{Resultados}

Se encontraron 418 muestras positivas a Eimeria spp entre las 478 muestras analizadas (87.5\%). El promedio de ooquistes por gramo de heces (OPGH) fue de 24 017, con un intervalo de confianza (IC) de 7534 y un rango de 50 a 1202400 OPGH.

Las mayores frecuencias de infecciones fueron debidas a $E$. lamae (60.4\%) y $E$. macusaniensis (50.4\%) (Cuadro 1); asimismo, el mayor porcentaje de crías infectadas se presentó en animales de 31 a 75 días de edad, y las mayores cargas parasitarias se observaron en el grupo etario de 46-60 días (Cuadro 2).

$\mathrm{Al}$ analizar la especie de Eimeria con la edad de los animales, se encontró que las crías se infectan muy tempranamente con E. lamae (41.7\%). Infecciones con E. ivitaensis fueron observadas en tasas bajas en todos los estratos etarios (Cuadro 3).

En el 28.2, 28.2 y 11.2\% de las muestras positivas se detectaron infecciones dobles, triples y cuádruples de Eimeria, respectivamente. En las asociaciones dobles predominó la coexistencia de E. lamae y E. alpacae (11.0\%), seguida de la asociación de E. lamae y E. macusaniensis (8.4\%). En las asociaciones triples predominó la coexistencia de E. alpacae, E. lamae y E. macusaniensis (10.8\%), seguida de E. punoensis, E. alpacae y E. lamae (5.7\%), mientras que en la presencia simultánea de cuatro especies de eimerias predominó la asociación de E. punoensis, E. alpacae, E. lamae y E. macusaniensis (9.8\%). Estas asociaciones no variaron entre grupos etarios, aunque se debe indicar que las combinaciones de E. lamae y E. macusaniensis se presentaron mayormente en el estrato etario de 61-75 días de edad.

En relación al análisis de riesgo multivariado para la presencia de eimerias en heces, se observó que las crías de 61 a 75 días de edad [odds ratio de 2.96 (I.C 1.495.87)] tuvieron un mayor riesgo de presentar ooquistes de eimeria en heces en relación al resto de animales, mientras que las variables separación de crías y el rango de edad de 130 constituyeron factores de protección (Cuadro 4). Las demás variables (raza, sexo, pre- 
Cuadro 1. Prevalencia de Eimeria, según especies, en 478 crías de alpaca del Centro de Investigación y Producción (CIP - La Raya) de la Universidad Nacional del Altiplano de Puno. 2008

\begin{tabular}{lcc}
\hline \multirow{2}{*}{ Especie } & \multicolumn{2}{c}{ Muestras positivas } \\
\cline { 2 - 3 } & $\mathrm{N} .^{\circ}$ & $\%$ \\
\hline E. lamae & 289 & 60.4 \\
E. macusaniensis & 210 & 50.4 \\
E. alpacae & 218 & 45.6 \\
E. punoensis & 142 & 30.0 \\
E. ivitaensis & 26 & 6.2 \\
\hline Total & 418 & 87.5 \\
\hline
\end{tabular}

Cuadro 2. Ooquistes de Eimeria spp por gramo de heces (OPGH) en crías de alpacas, según edad, del Centro de Investigación y Producción (CIP - La Raya) de la Universidad Nacional del Altiplano de Puno. 2008

\begin{tabular}{ccccc}
\hline \multirow{2}{*}{ Edad (días) } & \multicolumn{2}{c}{ Muestras positivas } & \multicolumn{2}{c}{ OPGH } \\
\cline { 2 - 5 } & N. ${ }^{\circ}$ & $\%$ & Promedio & Rango \\
\hline $1-30$ & $12 / 24$ & 50.0 & 17,216 & $50-103,650$ \\
$31-45$ & $76 / 82$ & 92.7 & 28,502 & $50-334,800$ \\
$46-60$ & $122 / 144$ & 84.7 & 34,372 & $50-1202,400$ \\
$61-75$ & $172 / 183$ & 94.0 & 16,564 & $50-387,600$ \\
$76-90$ & $36 / 45$ & 80.0 & 17,336 & $100-250,750$ \\
\hline Total & $418 / 478$ & 87.5 & & \\
\hline
\end{tabular}

Cuadro 3. Frecuencia porcentual de muestras positivas a Eimeria, según el grupo etario, en crías de alpacas del Centro de Investigación y Producción (CIP - La Raya) de la Universidad Nacional del Altiplano de Puno. 2008

\begin{tabular}{ccccccc}
\hline $\begin{array}{c}\text { Edad } \\
\text { (días) }\end{array}$ & $\begin{array}{c}\text { Muestras } \\
(\mathrm{n})\end{array}$ & $\begin{array}{c}E . \\
\text { punoensis }\end{array}$ & $\begin{array}{c}E . \\
\text { alpacae }\end{array}$ & $\begin{array}{c}E . \\
\text { lamae }\end{array}$ & $\begin{array}{c}\text { E. } \\
\text { macusaniensis }\end{array}$ & $\begin{array}{c}E . \\
\text { ivitaensis }\end{array}$ \\
\hline $1-30$ & 24 & 8.3 & 16.6 & 41.7 & 4.2 & 4.2 \\
$31-45$ & 82 & 26.8 & 39.0 & 64.6 & 15.9 & 1.2 \\
$46-60$ & 144 & 31.9 & 45.1 & 66.7 & 43.1 & 9.0 \\
$61-75$ & 183 & 33.9 & 54.6 & 60.1 & 65.6 & 4.9 \\
$76-90$ & 45 & 22.2 & 37.8 & 44.4 & 31.1 & 4.4 \\
\hline
\end{tabular}


Cuadro 4. Eimeriosis en crías de alpacas. Análisis univariado y multivariado para factores de riesgo de infección por coccidia (478 animales muestreados y 418 positivos)

\begin{tabular}{|c|c|c|c|c|c|c|}
\hline \multirow{2}{*}{ Variables } & \multicolumn{2}{|c|}{ Animales } & \multicolumn{2}{|c|}{ Análisis univariado } & \multicolumn{2}{|c|}{ Análisis multivariado } \\
\hline & Total & Positivos & $\mathrm{OR}^{1}$ & $\mathrm{P}$ & OR & $\mathrm{P}$ \\
\hline \multicolumn{7}{|l|}{ Edad (días) } \\
\hline$\geq 30$ & 24 & $\begin{array}{c}12 \\
(50.0 \%)\end{array}$ & 1 & \multirow{2}{*}{$<0.05$} & 1 & \multirow[b]{2}{*}{$<0.05$} \\
\hline$<30$ & 454 & $\begin{array}{c}406 \\
(89.4 \%)\end{array}$ & $\begin{array}{c}0.11 \\
0.05-0.27\end{array}$ & & $\begin{array}{c}0.19 \\
0.08-0.48\end{array}$ & \\
\hline $31-45$ & 82 & $\begin{array}{c}76 \\
(92.7)\end{array}$ & 1 & \multirow{2}{*}{ NS } & & \\
\hline$<31->45$ & 396 & $\begin{array}{c}342 \\
(83.3 \%)\end{array}$ & $\begin{array}{c}2.0 \\
0.83-4.81\end{array}$ & & & \\
\hline $46-60$ & 144 & $\begin{array}{c}122 \\
(84.7 \%)\end{array}$ & 1 & \multirow{2}{*}{ NS } & & \\
\hline$<46->60$ & 364 & $\begin{array}{c}296 \\
(81.3 \%)\end{array}$ & $\begin{array}{c}0.71 \\
0.40-1.25\end{array}$ & & & \\
\hline $61-75$ & 183 & $\begin{array}{c}172 \\
(93.9 \%)\end{array}$ & 1 & \multirow{2}{*}{$<0.05$} & 1 & \multirow{2}{*}{$<0.05$} \\
\hline$<60->75$ & 295 & $\begin{array}{c}246 \\
(83.4 \%)\end{array}$ & $\begin{array}{c}3.11 \\
1.57-6.16\end{array}$ & & $\begin{array}{c}2.49 \\
1.22-5.04\end{array}$ & \\
\hline $76-90$ & 45 & $\begin{array}{c}36 \\
(80.0 \%)\end{array}$ & 1 & \multirow{2}{*}{ NS } & & \\
\hline$<76->90$ & 433 & $\begin{array}{c}382 \\
(88.2 \%)\end{array}$ & $\begin{array}{c}0.53 \\
0.24-1.17\end{array}$ & & & \\
\hline \multicolumn{7}{|c|}{ Estratificación etaria } \\
\hline Sin & 87 & $\begin{array}{c}84 \\
(96.5 \%)\end{array}$ & 1 & \multirow{2}{*}{$<0.05$} & 1 & \multirow{2}{*}{$<0.05$} \\
\hline Con & 391 & $\begin{array}{c}334 \\
(85.4 \%)\end{array}$ & $\begin{array}{c}0.21 \\
0.06-0.84\end{array}$ & & $\begin{array}{c}0.2 \\
0.08-0.48\end{array}$ & \\
\hline \multicolumn{7}{|l|}{ Bofedales } \\
\hline Presencia & 127 & $\begin{array}{c}103 \\
(81.1 \%)\end{array}$ & 1 & \multirow{2}{*}{$<0.05$} & 1 & \multirow{2}{*}{ NS } \\
\hline Ausencia & 351 & $\begin{array}{c}315 \\
(89.7 \%)\end{array}$ & $\begin{array}{c}0.5 \\
0.27-0.86\end{array}$ & & $\begin{array}{c}0.75 \\
0.41-1.38\end{array}$ & \\
\hline \multicolumn{7}{|c|}{ Ubicación de la “punta” } \\
\hline Zonas altas & 46 & $\begin{array}{c}39 \\
(84.7 \%)\end{array}$ & 1 & \multirow{2}{*}{ NS } & & \\
\hline Zonas bajas & 432 & $\begin{array}{c}379 \\
(87.7 \%)\end{array}$ & $\begin{array}{c}0.76 \\
0.31-0.82\end{array}$ & & & \\
\hline \multicolumn{7}{|l|}{ Sexo } \\
\hline Macho & 234 & $\begin{array}{c}201 \\
(85.8 \%)\end{array}$ & 1 & \multirow{2}{*}{ NS } & & \\
\hline Hembra & 244 & $\begin{array}{c}217 \\
(88.9 \%)\end{array}$ & $\begin{array}{c}0.75 \\
0.44-1.30\end{array}$ & & & \\
\hline \multicolumn{7}{|l|}{ Raza } \\
\hline Huacaya & 417 & $\begin{array}{c}364 \\
(87.2 \%)\end{array}$ & 1 & \multirow{2}{*}{ NS } & & \\
\hline Suri & 61 & $\begin{array}{c}54 \\
(88.5 \%)\end{array}$ & $\begin{array}{c}0.89 \\
0.38-2.05\end{array}$ & & & \\
\hline
\end{tabular}


sencia de bofedales, crianza con estratificación etárea, ubicación de la "punta”) no fueron significativas en el análisis de riesgo (Cuadro 4).

\section{Discusión}

El presente estudio demuestra que el 87.5\% de crías de alpacas menores de 90 días de edad fueron positivas a Eimeria spp. Esta alta prevalencia es similar a los resultados hallados por otros autores nacionales en crías de alpacas (Guerrero et al., 1970b; Romero, 1992; Gallegos, 1994), en crías de llamas (Pelayo, 1973) y en crías de vicuñas (Salcedo, 1990). La alta prevalencia puede estar influenciada por una serie de factores que involucra presencia de animales portadores (adultas o tuis), contaminaciones de pasturas y letrinas, temperatura y humedad adecuadas para la esporulación de ooquistes, y época del año, así como por el tipo de manejo durante la parición, destete y esquila (Ameghino y DeMartini, 1991; Leguía, 1991).

El estudio muestra una mayor prevalencia de ooquistes en crías de 61 a 75 días de edad (94\%), seguido del estrato etario de 31 a 45 días (92.7\%). Estos resultados corroboran resultados anteriores (Guerrero et al., 1970b; Romero, 1992), donde además se indica que las crías eliminan ooquistes a partir de la segunda semana de edad (Leguía y Casas, 1999). Las infecciones tempranas en las crías sugieren exposiciones en los días posteriores al nacimiento y, quizás, exacerbadas por amamantamientos en condiciones insalubres o al inicio prematuro del consumo de pastos. Esta situación permitiría que las primeras crías infectadas, al inicio de la campaña de parición, actúen como animales amplificadores, eliminando grandes cantidades de ooquistes que contaminan las pasturas, incrementando las posibilidades de infecciones y reinfecciones, sobre todo para las crías que nacen posteriormente, especialmente en los meses más húmedos y temperados (febrero/marzo) (Rosadio et al., 2010).
Las especies de coccidia más prevalentes en el estudio fueron $E$. lamae (60.4\%) y E. macusaniensis (58.4\%) (Cuadro 1), similar a lo indicado por Gallegos (1994), pero discordantes con resultados de otros estudios (Guerrero, 1970b; Romero, 1992). Estas discrepancias podrían explicarse por diferencias climatológicas (humedad, temperatura, tiempo de radiación solar), manejo (separación etaria, rotación de dormideros, etc.) entre estudios. No obstante, e independientemente de las altas prevalencias, se podría considerar a estas especies dentro de los principales agentes patogénicos involucrados en el desarrollo de cuadros entéricos fatales para las crías de alpacas (Rosadio y Ameghino, 1994; Leguía 1999; Cebra et al., 2003).

Los resultados del estudio demuestran no solamente la elevada prevalencia de eimeriosis, sino también los continuos y progresivos ciclos de contaminaciones de las pasturas. Las elevadas tasas infectivas detectadas tienden a explicar algunas patologías comúnmente observadas en el centro experimental bajo estudio, asociadas a continuos procesos diarreicos y muertes súbitas de animales atribuidas a la enterotoxemia. Las elevadas tasas y cargas parasitarias, especialmente de E. macusaniensis, evidencian igualmente la probabilidad de observar infecciones prepatentes agresivas en animales de corta edad que podrían predisponer a coinfecciones clostridiales y terminar desarrollando el complejo enterotoxémico (Rosadio et al., 2010).

El estudio mostró que el $67.7 \%$ de las 418 muestras positivas presentaban infecciones múltiples, con dos a cuatro especies de Eimeria, aspecto que ha sido demostrado en estudios previos (Guerrero et al., 1970b; Hidalgo y Cordero, 1999). La presencia de infecciones simultáneas evidencia una probable patología sinérgica, particularmente entre las especies que se multiplican en vellosidades intestinales maduras (E. punoensis, E. alpacae y E. lamae) con aquellas como $E$. macusaniensis y E. ivitaensis que lo hacen 
en el epitelio de glándulas crípticas de la lámina propia (Rosadio y Ameghino, 1994; Palacios et al., 2006). En estas asociaciones llama la atención la presencia constante de $E$. lamae y E. macusaniensis en las infecciones dobles, triples y cuádruples.

El análisis de riesgo multivariado indicó que el rango etario de 61-75 días de edad fue un factor de riesgo asociado a la infección por eimerias, mientras que el rango etario de 1-30 días y la separación o estratificación por edades fueron factores de protección. Esto puede ser debido a la existencia, a los dos meses después de la parición, de una mayor población de crías susceptibles, efecto multiplicador de las primeras poblaciones (crías de 1-30 días de edad) al inicio de la campaña y/o estadios prepatentes de algunas de las especies de Eimeria que afectan a los camélidos (Melo y Hurtado, 1985; Leguía y Casas, 1999). La separación de las crías en grupos etarios dentro de la "punta" de parición aparentemente tuvo un efecto protector para la eliminación de ooquistes en las heces cuando se comparó con las "puntas" que no practicaron este tipo de separación etaria. La presencia simultánea de crías de distintas edades permitiría la convivencia entre animales amplificadores de grandes cantidades de ooquistes y de crías altamente susceptibles, manteniendo de esta forma, un activo ciclo infectivo (Cuadro 4).

La alta tasa de infecciones mixtas con elevadas cargas de OPGH durante los primeros tres meses de vida corrobora el potencial patogénico de posibles interacciones de dos o más especies de coccidia en etapas neonatales de la crianza de alpacas (Cuadros 2 y 3). El ciclo infectivo de las especies de coccidia persiste en el medio por diversos factores, pero fundamentalmente por la presencia de animales crónicamente infectados (Leguía y Casas, 1999; Cebra et al., 2007; Cafrune et al., 2009) y el uso continuo y sin descanso de "canchas" contaminadas en la época de parición. En este contexto, los animales portadores contribuirían a una perma- nente contaminación de la pastura con ooquistes favorecido por un estrés de manejo en que se encuentran las alpacas gestantes. Las futuras madres, desde mediados de noviembre, se exponen a actividades estresantes que se inician con la esquila anual, seguido de largas caminatas hacia las "canchas" de pariciones (inicios de diciembre), además de experimentar la parición y empadre casi inmediato a que son sometidos anualmente (Rojas 1990; Ameghino y DeMartini, 1991).

Todas estas prácticas de manejo pueden llevar a la pérdida temporal de la inmunidad innata en estos animales y ocasionar un incremento en la eliminación de ooquistes y contaminación de las pasturas. Esto ocasionaría infecciones tempranas en las nuevas poblaciones de crías que a su vez actuarían como replicadoras de ooquistes para el resto de crías hasta el término de la campaña de parición (marzo). El ciclo infectivo se completaría con el microclima favorable para el desarrollo, viabilidad y maduración de los ooquistes propios de los meses más húmedos y templados (diciembre a marzo), que coincide con la parición de las alpacas en los Andes peruanos (Romero, 1992; Gallegos, 1994; Leguía y Casas,1999). Las potenciales interacciones patológicas de infecciones múltiples, particularmente las combinaciones entre eimerias epiteliotrópicas intestinales maduras y E. macusaniensis, merecen ser estudiadas detenidamente.

\section{Conclusiones}

- El 87.5\% de la población de crías de alpaca estuvo infectada por especies de Eimeria, especialmente por E. lamae (60.4\%) y E. macusaniensis (50.4\%).

- El mayor porcentaje de crías infectadas se presentó en animales de 31-75 días de edad, y las mayores cargas parasitarias se observaron en el grupo etario de 46-60 días.

- Las crías se infectan tempranamente con E. lamae. 
- $\quad$ En el 28.2, 28.2 y 11.2\% de las muestras positivas se detectaron infecciones dobles, triples y cuádruples.

- El rango etario de 61-75 días fue considerado como factor de riesgo, en tanto que el rango etario de 1-30 días y la separación de crías por edades fueron considerados como factores de protección para la presencia de ooquistes de Eimeria en heces.

\section{LiTERATURA CITADA}

1. Ameghino E, DeMartini J. 1991. Mortalidad en crías de alpacas. Lima: IVITA, RERUMEN. 128 p.

2. Cafrune MM, Marín RE, Rigalt FA, Romero SR, Aguirre DH. 2009. Prevalence of Eimeria macusaniensis and Eimeria ivitaensis in South American camelids of Northwest Argentina. Vet Parasitol 162: 338-341.

3. Cebra CK, Mattson DE, Baker, RJ, Sonn RJ, Dearing PL. 2003. Potential pathogens in feces from unweaned llamas and alpacas with diarrhea. J Am Vet Med Assoc 223: 1806-1808.

4. Cebra CK, Valentine BA, Schlipf JW, Bildfell RJ, McKenzie E, Waitt LH, Heidel JR, et al. 2007. Eimeria macusaniensis infection in 15 llamas and 34 alpacas. J Am Vet Med Assoc 230: 94-100.

5. [FAO] Food and Agriculture Organization of the United Nations. 2005. Situación actual de los camélidos sudamericanos en el Perú. Proyecto de cooperación técnica en apoyo a la crianza y aprovechamiento de los camélidos sudamericanos en la Región Andina. TCP/ RLA/2914. Rome: FAO. 62p.

6. Gallegos D. 1994. Parasitismo gastrointestinal en crías de alpacas. Tesis de Médico Veterinario. Lima: Facultad de Medicina Veterinaria, Univ Nacional Mayor de San Marcos. 37 p.

7. Guerrero C. 1967. Coccidia (Protozoa: Eimeriidae) of the alpacas Lama pacos. J Protozool 14: 613-616.
8. Guerrero C, Hernández J, Alva J. 1967. Coccidiosis en alpacas. Bol Ext IVITA, Perú 2: 66-67.

9. Guerrero C, Alva J, Bazalar H, Tabacci L. 1970a. Infección experimental de alpacas con Eimeria lamae. Bol Ext IVITA, Perú 4: 79-83.

10. Guerrero C, Alva J, Leguía G Bazalar H. 1970b. Prevalencia de coccidias (Protozoa: Eimeriidae) en alpacas ( Lama pacos). Bol Ext IVITA 4: 84-90.

11. Hidalgo MR, Cordero del Campillo M. 1999. Coccidiosis. En: Cordero del Campillo M y Rojo M (ed). Parasitología veterinaria. Madrid: Mc Graw HillInteramericana. p 195-197.

12. Leguía G. 1991. The epidemiology and economic impact of llama parasites. Parasitol Today 7: 54-56.

13. Leguía G Casas E. 1999. Enfermedades parasitarias y atlas parasitológico de camélidos sudamericanos. Lima: Ed La Mar. $930 \mathrm{p}$.

14. Melo M, Hurtado E. 1985. Infestación parasitaria en alpacas desde el nacimiento hasta el destete. Rev Inv Camélidos Sud ALLPAK'A 1(2): 78-86.

15. Palacios $C$, Perales $R$, Chavera A, López M, Braga W, Moro M. 2006. Eimeria macusaniensis and Eimeria ivitaensis co-infection in fatal cases of diarrhea in young alpacas (Lama pacos) in Peru. Vet Rec 158: 344-345.

16. Pelayo A. 1973. Prevalencia de coccidias. (Protozoa: Eimeridae) en llamas (Lama glama). Tesis de Bachiller. Lima: Facultad de Veterinaria, Univ Nacional Mayor de San Marcos. 30 p.

17. Rojas M. 1990. Parasitismo de los rumiantes domésticos. Terapia, prevención y modelo para su aprendizaje. Lima: Ed Maijosa. 383 p.

18. Romero M. 1992. Prevalencia y carga parasitaria de Eimeria sp en crías de alpacas. Tesis de Bachiller. Lima: Facultad de Medicina Veterinaria, Univ Nac Mayor de San Marcos. 28 p.

19. Rosadio R, Ameghino E. 1994. Coccidial infections in neonatal Peruvian alpacas. Vet Rec 135: 459-460. 
20. Rosadio R, Londoñe P, Perez D, Castillo H, Veliz A, Llanco L, Yaya K, Maturrano L. 2010. Eimeria macusaniensis associated lesions in neonate alpacas dying from enterotoxemia. Vet Parasitol 168: 116-120.

21. Salcedo C, Gálvez H. 1990. Estudio comparativo de la infestación por pará- sitos gastrointestinales en vicuñas en semicautiverio. En: Resúmenes Congreso Nacional de Ciencias Veterinarias. Cusco, Perú. p 18.

22. Wheeler CJ. 1995. Evolution and present situation of the South American Camelidae. Biol J Linn Soc 54: 271-295. 